\title{
Film und Wissenschaft: Übergänge, Zusammenhänge und Parallelitäten. Eine Einführung
}

Iris Ritzmann, Hans-Konrad Schmutz und Eberhard Wolff

Kein Medium hat das kollektive Wahrnehmen und den Denkstil des letzten Jahrhunderts so stark geprägt wie der Film. Grund genug, dass sich auch die Wissenschafts- und speziell die Medizingeschichte dieses Themas annimmt. Diese Ausgabe des «Gesnerus» versammelt als Themenheft historische Beiträge zum komplexen Verhältnis, das zwischen Film und Medizin sowie Naturwissenschaften herrschte. Einige von ihnen basieren auf Vorträgen der Jahrestagung zu diesem Thema, welche die Schweizerische Gesellschaft für Geschichte der Medizin und der Naturwissenschaften (SGGMN) 2006 durchgeführt hat.

In den letzten Jahren nahmen Naturwissenschaftler zunehmend Anteil an der Produktion von Unterhaltungsfilmen. So brachten Meeresbiologen Disneys kleinem «Nemo» das korrekte Schwimmen eines Clownfisches bei ${ }^{1}$. Regelmässig treffen sich Wissenschaftler mit Filmleuten am renommierten American Film Institute in Hollywood ${ }^{2}$. Drehbuchautoren und Biologen wollen gemeinsam das ramponierte Image der Wissenschaft in der Unterhaltungskultur korrigieren. Wissenschaftler erschienen dort nur zu oft «labcoated» und «frizzy-haired $»^{3}$, bald bösartig wie Frankenstein, bald schrullig wie der «Nutty Professor» Jerry Lewis, jedoch immer als ein gesellschaftlicher Problemfall. In einer Welt, in der grundlegende wissenschaftliche

1 Alison, A., «The Fabulous Fish Guy», Nature 427 (2004) 672f.

2 Knight, J., «Hollywood or Bust», Nature 430 (2004) 720-723.

3 Frayling, Christopher, Mad, Bad and Dangerous? The Scientist and the Cinema (London 2004) 32.

Iris Ritzmann, Medizinhistorisches Institut und Museum der Universität Zürich, Hirschengraben 82, CH-8001 Zürich (iritz@mhiz.uzh.ch).

Hans-Konrad Schmutz, Naturmuseum, Museumsstrasse 52, CH-8400 Winterthur; Anthropologisches Institut und Museum der Universität Zürich (hanskonrad.schmutz@win.ch).

Eberhard Wolff, Medizinhistorisches Institut und Museum der Universität Zürich, Hirschengraben 82, CH-8001 Zürich (eberhard.wolff@mhiz.uzh.ch). 
Erkenntnisse wie zum Beispiel die Abstammung des Menschen a priori in Frage gestellt werden und folglich auch die politische Akzeptanz der wissenschaftlichen Erkenntnisse zur Diskussion steht ${ }^{4}$, hat die Akademia das Bedürfnis, weniger als verrückte Wissenschafter denn als kreditwürdige Troubleshooter der Gesellschaft dazustehen.

Bilden sich im Unterhaltungsfilm aber unsere Vorstellungen von der Wissenschaft 1:1 ab? Wie werden wissenschaftliche Erkenntnisse über das Medium Film in eine breite Öffentlichkeit transportiert? Hat dies Rückwirkungen auf die Wissenschaft? Die Aufsätze dieses Themenheftes suchen historische Antworten auf solche und weitergehende Fragen. Diese Aufsatzsammlung vereint eher überblicksartige Beiträge wie diejenigen von Jakob Tanner, Francesco Panese und Christian Bonah/Anja Laukötter mit eher an Einzelbeispielen orientierten Studien wie die von Philipp Osten und Mireille Berton. Jeder Beitrag hat seinen eigenen Schwerpunkt, doch im Unterschied zu vielen anderen Themenheften von Fachzeitschriften bietet die Summe der Beiträge dieser Ausgabe mehr als einen bunten Strauss unterschiedlicher Ansätze zu einer Vielfalt von Themen. Die Beiträge dieses Heftes besitzen eine ausgesprochene Kohärenz. Sie überschneiden sich, sie ergänzen sich und schaffen Querbezüge. Anders als in klassischen Einleitungen dieser Art können, ja dürfen die vorliegenden Beiträge deshalb nicht einzeln nacheinander skizziert werden, sondern anhand ihrer gemeinsamen, übergreifenden Fragestellungen. Diese Art der Einleitung soll helfen, jeden einzelnen Beitrag und seine Hauptakzente im gesamten Themenheft zu verorten ${ }^{5}$.

Oberflächlich betrachtet sind Film und Wissenschaft zwei völlig unterschiedliche Sachen: «Die» Wissenschaft ist ein überaus komplexes Verfahren zur Erkenntnisgewinnung, der Film ist am Anfang nicht mehr als eine Visualisierungstechnik. Auch die Kontaktzonen zwischen Film und Wissenschaft fallen zunächst in zwei Bereiche auseinander:Zum einen nutzten etwa die Naturwissenschaften und die Medizin das Medium Film bereits kurz nach seiner Entwicklung für die Dokumentation von Experimenten oder Befunden. Als sich der Film später auch als Unterhaltungsmedium etablierte, boten Naturwissenschaft und Medizin zum anderen ein häufiges und interessantes Sujet für dieses Unterhaltungsmedium und etablierten damit ein völlig anderes Kontaktfeld von Film und Wissenschaft. Doch das oberflächlich dichotomische Bild zerfällt bei genauerer Betrachtung schnell, und

4 Miller, J. D./E. C. Scott et al., «Public Acceptance of Evolution», Science 313 (2006) $765 f$.

5 Um nicht den Eindruck zu erwecken, die Herausgeber wollten sich mit fremden Federn schmücken, sei betont, dass in dieser Einleitung durchgängig Aussagen aus den folgenden Beiträgen referiert werden. Ihre Synthese allerdings verantworten die Herausgeber selbst. 
genau in diesem Sinne nehmen uns die Beiträge dieses Themenheftes auf eine analytische Reise, in der die Grenzen zwischen Film und Wissenschaft sowie ihren unterschiedlichen Kontaktzonen bis zur Unkenntlichkeit verschwimmen sowie komplexe und ungeahnte neue Bezüge entstehen.

Dies beginnt bereits bei den Filmen, die zur reinen Dokumentation wissenschaftlicher Phänomene erstellt wurden. So machen die Beiträge von Panese und Tanner klar, dass sich Wissenschaft und Medizin mit dem Film nicht einfach nur eines praktischen technischen Dokumentationsmittels bedienten. Der Film ermöglichte, etwa mit Zeitlupe und Zeitraffer, mikroskopischen Filmaufnahmen oder gar dem Röntgen, Dinge zu sehen, die ohne diese Technik nicht oder schwerer zu erkennen, zu entdecken oder zu verstehen waren. Der Film konnte so das Unsichtbare sichtbar machen. Mit dieser epistemischen, erkenntnisfördernden Funktion wurde der Film zum integralen Teil der Experimentalanordnung, der medizinischen Diagnose oder gar der Therapie. Das Medium wirkte auf diese Weise zurück auf die Inhalte der wissenschaftlichen Praxis und veränderte sie. Die «visuelle Rhetorik» des Films konnte und kann den Blick des Betrachters führen, Schwerpunkte setzen, der Medizin ein erfolgreiches Image oder Krankheiten einen objektiven Anschein geben. Diese Eigenschaft hat der Film in Naturwissenschaft und Medizin nach Panese gemein mit seinen Vorläufern, etwa Bildreihen. Umso mehr gilt dies für die Gegenwart mit ihrem «medizinischen Okularozentrismus», zum Beispiel in Form von optischen Verfahren jenseits des Films wie videogestützten endoskopischen Operationstechniken. Der medizinische Film ist in diesem Sinne nur ein Beispiel für viele Varianten einer visuellen Ausformung des Körpers, die jeweils ihre eigenen Bilder, Vorstellungen vom Körper und Umgangsweisen mit ihm entstehen lassen.

Die Trennlinie zwischen dem wissenschaftlichen Film als Dokumentationsmedium und dem Film als Verbreitungsmedium wurde bereits in dem Moment unscharf, als wissenschaftliche Filme innerakademisch für die Lehre sowie die Aus- und Fortbildung eingesetzt wurden. Aus dieser Perspektive gesehen ist der nächste Schritt zum Film als Medium der Wissenschaftspopularisierung in die ausserakademische Öffentlichkeit nur noch ein gradueller. Nahezu alle Beiträge dieses Themenheftes befassen sich mit dem überaus hybriden Genre des medizinischen Aufklärungsfilms, der sich speziell in Deutschland, moralisch und cineastisch aufbereitet, in den 1920er Jahren unter dem Titel «Kulturfilm» etablierte. Sind diese «Kulturfilme» noch Popularisierungen von Wissenschaft oder bereits Teil einer Unterhaltungsindustrie, fragt Tanner und plädiert dafür, anstelle klarer Grenzen ein Kontinuum zwischen wissenschaftlicher Kommunikation und Populärkultur zu sehen. Bonah und Laukötter sehen diese Stellung zwischen Wissenschaft und 
Unterhaltung in ihrem Beitrag sogar als das zentrale Merkmal der Aufklärungsfilme und beschreiben es mit ihrer vergleichenden Perspektive als internationales Phänomen. Darauf aufbauend interpretieren sie diese Filme als «boundary objects» und skizzieren das analytische Potential, welches künftige historische Analysen medizinischer Filme aus dieser Perspektive entwickeln könnten. Das gleiche Phänomen illustriert Osten am konkreten Beispiel anhand des Films «Krüppelnot und Krüppelhilfe». Der Autor zeigt, wie dieser Film zunächst als nüchterner «Lehrfilm» begonnen und schliesslich mittels szenischer Dramaturgie und emotionalen Plots als «Kulturfilm» mit Unterhaltungselementen fertiggestellt wurde. Ein anderes Beispiel Ostens, die filmische Dokumentation einer Amputation durch den Berliner Chirurgen Ernst von Bergmann, mutierte gar vom innermedizinischen Dokumentationsfilm zur Jahrmarktsattraktion.

Medizinische Aufklärungsfilme sind mehr als ein Überschneidungsfeld von akademischer Medizin und Unterhaltungskultur. Sie sollten immer auch auf das gesundheitliche Denken und Handeln ihrer Zuschauer einwirken. «Kulturfilme» waren damit auch Produkte gesundheitspolitischer Agenten und Interessengruppen, womit ein dritter wesentlicher Akteur hinzutritt. Osten fügt dem Film «Krüppelnot und Krüppelhilfe» aus diesem Grund auch die weitere Eigenschaft «Propagandafilm» hinzu, der gleichzeitig auf das gesundheitliche Verhalten der Bevölkerung einwirken und ein umstrittenes Gesetzesvorhaben unterstützen sollte - ganz zu schweigen davon, dass er auch dem Fundraising dienen sollte. Im Sinne von Bonah und Laukötter wurde der Film damit noch mehr zu einem «boundary object». Und was Wunder, dass auch personell die Grenzen verschwimmen. Verantwortlich für die auffällige Gleichförmigkeit des deutschen Kulturfilms war eine über Jahrzehnte weitgehend konstante Gruppe von Personen, die als Vertreter von Staat, Medizin und Kinogeschäft Einfluss auf dessen Gestaltung nahm. Entsprechend fragt Osten auch rhetorisch: Waren es die Sachverständigen, die hier Politik gestalteten, oder kontrollierten die leitenden Regierungsräte die in ihrem Gremium vertretenen Mediziner und Pädagogen? Die Antwort ist, dass diese Unterscheidung nicht weiterführt, weil die Beteiligten in doppelten oder wechselnden Rollen agierten. Die Gleichförmigkeit des Kulturfilms wurde von einem kaum entwirrbaren staatlich-medizinischpädagogisch-cineastischen (und zeitweise auch militärischen) Einflusskomplex geschaffen, der klassische Grenzziehungen verschwimmen lässt.

Neben dem gesundheitspropagandistischen Kulturfilm entstanden im frühen 20. Jahrhundert aber auch Filme, die neue (natur-)wissenschaftliche Erkenntnisse einfach nur an ein interessiertes Publikum weitergeben wollten. Man kann das Prinzip populärwissenschaftlicher Filme als Vermittlung 
vereinfachter wissenschaftlicher Inhalte durch ein Massenmedium an ein breites Publikum verstehen. In dieser Sichtweise ist das Medium «Film» lediglich der Transporteur von Inhalten, und dies ausschliesslich in eine Richtung. Anhand von zwei Beispielen filmischer Wissenschaftspopularisierung illustriert Tanner jedoch, dass wissenschaftsdokumentarische Filme mehr sind. Der Versuch unter Wissenschaftlern, in den 1920er Jahren Populationsgenetik mit dem Medium Film in der Öffentlichkeit bekannt zu machen, liess inhaltliche Differenzen zwischen Wissenschaftlern über den Forschungsgegenstand selbst (nicht nur seine Popularisierung!) aufbrechen, die sonst nicht bewusst geworden wären. Der Popularisierungsversuch hatte so einen «theoretisch klärenden Effekt» auf den Gegenstand selbst. Die Popularisierung von Wissenschaft im Film zwingt nach Tanner also zur Vereinfachung und Anschaulichkeit, was zur Überprüfung der Hypothesen anregen und Ideen für neue experimentelle Beweisführung liefern kann. So hat auch der Film als Medium popularisierter Wissenschaft eine Rückwirkung auf die Inhalte der Forschung. Mehr noch: Nicht allein die Popularisierung als solche hat diesen Effekt, sondern ganz speziell auch das Medium Film mit seinen technischen Möglichkeiten. Ein Trickfilm, der die Relativitätstheorie einem breiten Publikum zugänglich machen sollte, vermochte diese Theorie mit virtuellen Beispielen und visuellen Formen zu plausibilisieren, die als wirkliches Experiment nie realisierbar gewesen wären. Das filmische Spiel mit theoretischen Aussagen (ob popularisiert oder nicht) wirkt damit auf das Selbstverständnis der wissenschaftlichen Forschung zurück. Die neuen Bildmedien, schliesst Tanner daraus, böten somit auch die Chance, die kritische Selbstreflexivität wissenschaftlicher Praktiken zu erhöhen.

Dass sich die Relativitätstheorie gerade mit einer gleichzeitigen Innovation, dem Trickfilm, so gut illustrieren liess, sei nicht nur ein Zufall, sondern liege auch in Homologien zwischen der wissenschaftlichen Theoriekonstruktion und der filmischen Visualisierungslogik begründet. An dieser Stelle tut sich ein weiterer «roter Faden» auf, der sich durch mehrere der folgenden Aufsätze zieht. Die Beitragenden sehen allgemein deutliche Parallelitäten zwischen der Entwicklung des Mediums Film, speziell auch des Unterhaltungskinos, und Entwicklungen in verschiedenen Wissenschaftsbereichen, womit die Grenze zwischen Wissenschaft und Film eine weitere Stufe durchlässiger erscheint.

Auf eine dieser Parallelen weist Panese hin: Im Anti-Kurpfuscher-Film «Frauennot, Frauenglück» lässt sich die medizinische Rhetorik (der Gang zum Kurpfuscher bringt den Tod, das Vertrauen in die akademische Medizin bringt hingegen Glück) ohne Probleme mit der dramatischen Rhetorik des Spielfilms (Problem und Auflösung) deckungsgleich machen. Die 
Medizin bediente sich hier der gleichen Rhetorik wie das dramatische Filmgenre.

Noch um einiges deutlicher werden Parallelitäten im Beitrag von Berton. Die Autorin untersucht Verbindungen zwischen dem frühen Kino und den zeitgenössischen Psychowissenschaften. Mit dem Unterhaltungskino entwickelte sich ein Diskurs über die psychischen, neurologischen und emotionalen Wirkungen dieses ebenso neuen wie eindrucksvollen Mediums - ein Diskurs, auf den auch Osten in seinem Beitrag zu sprechen kommt. Allerdings machte dieser Diskurs nach Berton nicht einfach nur Anleihen bei den zeitgenössischen Psychowissenschaften und ihren Subjektivitätsmodellen, etwa dem «neurotischen» Subjekt im Geiste der Neurastheniedebatte, welches das Kino befördern würde. Für die Psychowissenschaften lieferte das Kino auch Modelle und Beispiele, etwa um Erinnerungsveränderungen zu untersuchen. Wiederholte Erinnerungen an aufwühlende Filmszenen konnten in die Nähe von psychischen Abnormalitätsphänomenen wie Halluzinationen oder Visionen gebracht werden. Das Kinophänomen, sich als Zuschauer ganz in eine Filmrolle hineinzuversetzen, wurde im Zusammenhang mit dem psychiatrischen Phänomen doppelter Identitäten oder kompletter Identitätswechsel, etwa im Rahmen des Somnambulismus, diskutiert. Die experimentelle Psychologie dieser Zeit brachte ihre Probanden in Laboratorien in unterschiedliche emotionale Zustände, um daraus Regeln von Normalität und Normierung abzuleiten. In einem ganz parallelen Sine wurde das Unterhaltungskino mit seinen emotionalen Wechselbädern als Laboratorium zur Erzeugung des modernen Subjekts verstanden. Der Dialog der Psychowissenschaften mit der Welt des Kinos hatte auf diese Weise einen eigenen Einfluss auf deren Theoriebildung. So lässt sich mit Berton fragen: Ist der Kinobesucher nicht ebenso ein Versuchsobjekt wie die Probanden in psychowissenschaftlichen Laboratorien? Oder mehr noch: Hat das Kino nicht mitgeholfen, eben jenes moderne Subjekt zu entwickeln, wie es die Psychowissenschaften taten?

Wenn der aufklärerisch-propagandistische «Kulturfilm» wie oben erwähnt eine mittlere Position im Kontinuum zwischen wissenschaftlicher Kommunikation und Populärkultur einnimmt, so ist der reine Unterhaltungsfilm, der von Wissenschaft handelt, an einem der Endpunkte angesiedelt. Beide gehen mit ihren dramaturgischen Elementen, Emotionen oder subtiler Erotik auf populäre Zuschauerbedürfnisse ein. Sind diese Bedürfnisse im Kulturfilm, so Osten, jedoch noch ein Mittel zum propagandistischen Zweck, so werden sie im Unterhaltungsfilm zum eigentlichen Programm. In der konkreten Aussage ist dieses Kontinuum allerdings eine fundamentale Bruchlinie: Während im propagandistischen «Kulturfilm» die medizinische 
«Wissenschaft» grundsätzlich in einem positiven, kreditwürdigen Licht erscheint, dominiert im Unterhaltungsfilm der 1920er Jahre der «mad scientist» das Bild. War dieser aber darauf angelegt, die Wissenschaft als solche zu diskreditieren - im Gegensatz zum Kulturfilm? Gerade dies verneint Tanner. Die Ansammlung von «mad scientists» à la Dr. Frankenstein und Dr. Rotwang in «Metropolis» ist für ihn Teil einer allgemeinen cineastischen Entwicklung der Zeit, nämlich der «Eskalation des Spektakulären». Der «verrückte Wissenschaftler» ist vor allem ein Angebot an das Filmpublikum, in eine emotionale Filmstory zu investieren, sich zeitweilig mit dem Bösen und dem Wissenden zu identifizieren und sich am Schrecklichen erfreuen zu können. Das Publikum nahm dieses Angebot offenbar gerne an, aber sein Bedürfnis nach fiktionaler Identifizierung musste keine eindeutige Entsprechung in der Realität haben. Wenn durchgedrehte Forscher auf der Leinwand wüteten, war die Diskreditierung der Wissenschaft demnach nicht das eigentliche Ziel - das mag immerhin eine kleine Beruhigung für die Wissenschaftler sein, die sich in den letzten Jahren im American Film Institute um das Image der Wissenschaft bemühen.

Kohärenz besitzen die Beiträge dieses Schwerpunktheftes schliesslich noch darin, dass sich das aktuelle Forschungsthema «Wissenschaft und Emotionen» wie ein roter Faden durch die Aufsätze zieht, ohne dass es aber den eigentlichen Mittelpunkt der Fragestellungen bildet. Das Themenheft hat auch alles andere als den Anspruch, das Forschungsfeld «Wissenschaft im Film - Film in der Wissenschaft» vollständig abzudecken. Eine mögliche Richtung weiterer Forschungen schlagen Bonah und Laukötter unter dem Stichwort «boundary objects» vor. Offensichtlich ist auch, dass die Forschung über innerwissenschaftlich eingesetzte Filme noch einige Zuwendung vertragen kann. Schliesslich wäre es ein lohnenswertes Unterfangen, der Rezeptions- und mehr noch der Wirkungsgeschichte des populären Wissenschaftsfilms genauer nachzugehen. Um nur ein Beispiel zu nennen: «Serengeti darf nicht sterben», der oscarprämierte Naturfilm des deutschen Fernseh-Zoologen Bernhard Grzimek von 1959, hat den Naturschutzgedanken in der breiten Bevölkerung zur Adenauerzeit wohl stärker als alle wissenschaftliche Feldforschung geprägt.

Die Jahrestagung, die diesem Themenheft zugrunde liegt, gab nicht nur wissenschaftshistorisch-analytischen Ansätzen ein Podium. Auf ihr kam mit dem Hollywood-Regisseur und Kubrick-vertrauten Alexander Singer sowie dem bekannten Schweizer Naturfilmer Andreas Moser auch die eher narrative Perspektive der Medienpraktiker zu Wort. Diese Perspektive greift der vorliegende Band mit zwei ausführlichen Rezensionen des langjährigen Fernsehjournalisten und Wissenschaftsredakteurs Peter Lippuner ebenfalls auf. 
Angesiedelt an der Nahtstelle zwischen Wissensproduktion und Unterhaltung ist der Film ein zentrales Forschungsfeld auch der historischen Wissenschaftsforschung. Die vorliegende Aufsatzsammlung soll einen Einblick in dieses hochspannende und aktuelle Forschungsfeld gewähren. 